\title{
Entre el ciberespacio y la calle: etnografiando grupos juveniles de calle en tiempos de distanciamiento físico
}

\author{
José SAnChez-García \\ Universitat Pompeu Fabra \\ jose.sanchez@upf.edu \\ 0000-0002-2880-7813
}

\author{
María Oliver \\ Universitat Pompeu Fabra \\ maria.olivert@upf.edu \\ 0000-0002-6396-9009
}

\author{
Juan Camilo Mansilla \\ Universitat Pompeu Fabra \\ juan.mansilla@upf.edu \\ 0000-0001-5856-2717
}

\author{
Nele Hansen \\ Universitat Pompeu Fabra \\ nele.hansen@upf.edu \\ 0000-0003-0832-5654
}

\author{
Carles Feixa \\ Universitat Pompeu Fabra \\ carles.feixa@upf.edu \\ 0000-0002-4874-1604
}

\section{Between cyberspace and the street: Conducting ethnography with youth street groups in times of physical distancing}

\begin{abstract}
RESUMEN
Analizamos los diferentes escenarios que afronta nuestro proyecto de investigación al decretarse la alerta sanitaria por Coronavirus, partiendo de un cuestionario realizado a 16 investigadores/as del proyecto TRANSGANG en el Magreb, el sur de Europa y las Américas. En primer lugar, analizamos el impacto de las medidas de distanciamiento físico sobre las relaciones sociales, en especial sobre los grupos juveniles de calle. En segundo lugar, apoyados en los diferentes contextos etnográficos del proyecto, estudiamos cómo se construyen las relaciones de confianza entre investigado-

res e informantes, las ventajas y límites que impone una etnografía (más o menos) híbrida, entre el ciberespacio y la calle. En tercer lugar, planteamos los desafíos que tiene el uso de tecnologías de comunicación en el trabajo etnográfico con grupos juveniles de calle, enmarcados en contextos sociopolíticos diversos. Por último, vemos cómo estos grupos juveniles se adaptan a las nuevas condiciones de control social, participando en formas de resistencia históricas (ilegalidad, economía formal, enfrentamientos con la policía). Concluimos que el uso de las comunicaciones a distancia con los miembros de grupos juveniles de calle, siendo una estrategia etnográfica útil para preservar el contacto, necesita de encuentros físicos previos donde (eventualmente) la con-
\end{abstract} fianza se construye.

PALABRAS CLAVE

Confianza; Confinamiento; Etnografía; Jóvenes; Grupos juveniles de calle; Tecnologías de comunicación.

\section{ABSTRACT}

We analyse the different scenarios that our research TRANSGANG had to encounter in the moment the health alert due to the pandemic Coronavirus was decreed. We base our analysis on a questionnaire carried out to 16 TRANSGANG researchers in the Maghreb, southern Europe and the Americas. First, we examine the impact of physical distancing measures on social relationships, especially on youth street groups. Second, taking into account the different ethnographic contexts of the project, we consider how relationships of trust are built in these times between researchers and informants, the advantages and limits imposed by a (more or less) hybrid ethnography, between cyberspace and the street. Third, we debate the challenges of the use of communication technologies in ethnographic work with street youth groups that are framed in diverse socio-political contexts. Finally, we comment on how these youth groups adapt to the new conditions of social control, participating in historical forms of resistance (illegality, formal economy, confrontations with the police). We conclude that the communication from the distance with members of street youth groups, being a useful ethnographic strategy to preserve contact, requires prior physical encounters where (eventually) trust is built.

\section{KEYWORDS}

Trust; Confinement; Ethnography; Youth; Youth street groups; Communication technologies. 


\section{Introducción}

A fines de febrero y mediados de marzo, el coronavirus comenzó a asaltar nuestra vida cotidiana convirtiéndose en el centro de atención de la producción científica mundial. Al mismo tiempo, la gestión de la pandemia también ha modificado investigaciones en curso y obligado a una "adaptación" acelerada para gestionarlas. Es el caso del proyecto TRANSGANG, centrado en la obtención de datos sobre la cotidianeidad de agrupaciones juveniles callejeras en doce ciudades del planeta: Barcelona, Madrid, Marsella y Milán en Europa; Chicago, San Salvador, Santiago de Cuba y Medellín en América; y Casablanca, Argel y el Gran Túnez en el Norte de África. Se trata de una investigación eminentemente etnográfica sobre la sociabilidad de grupos juveniles de calle y en especial de qué manera se producen procesos de mediación que mitiguen las estigmatizaciones y criminalizaciones que sufren. El foco en la mediación, básicamente una forma de interacción social física, la convierte en un tipo de investigación que exige el contacto cara a cara con las y los participantes para trabar confidencialidades que permitan abordar temas delicados y sensibles como las actividades de los grupos de calle y los conflictos que se producen tanto con otros grupos como con autoridades estatales (Feixa, Sánchez-García y Brisley, 2020). Los participantes en la investigación son jóvenes entre los 16 y los 29 años que muestran o han mostrado membresías en grupos juveniles de calle, más o menos organizados. Son miembros o ex miembros de los Latin Kings, DDT, trinitarios y jóvenes árabes en Europa; de las maras salvadoreñas, rastafaris o rockeros en Cuba; de los vendedores informales de las ciudades magrebíes y asociaciones, más o menos institucionalizadas, que trabajan con diferentes formas de activismo social para producir mediación natural (Feixa et al, 2019), como son los grupos dedicados a las culturas urbanas, el caso del grupo tunecino Debo en Túnez, y otras agrupaciones como casa Kolacho en Medellín, dedicada al trabajo con jóvenes en barrios marcados por la violencia.' Además, se han trabado relaciones con agentes que participan en el campo social de los grupos juveniles de calle, desde miembros de las fuerzas de seguridad a trabajadores sociales pasando por asociaciones no gubernamentales que trabajan con los colectivos investigados. Así, los diferentes trabajos de campo en cada una de las localizaciones se iniciaron durante el último trimestre de 2019, estando suficientemente avanzados el proceso de entrada al campo cuando se produjo el inicio de la pandemia y la aparición de las medidas para luchar contra el virus.

De alguna manera, esta aparición repentina ha obstaculizado el afianzamiento de relaciones de confianza entre participantes e investigadores establecida en los meses previos a la pandemia, imprescindibles para la comunicación entre las partes en una investigación que entiende la etnografía como una metodología². Así, la necesidad de adaptación a la nueva situación parecía obligar a un replanteamiento de la manera de realizar las tareas asociadas al trabajo de campo. Pero, ¿de qué manera? ¿Cómo han usado las/os jóvenes callejera/ os la comunicación virtual tanto entre miembros como con la/os propios investigadores? ¿Qué obstáculos se producen para una comunicación fluida virtual en investigaciones etnográficas dependientes de una relación de confianza como la que propone TRANSGANG?

Para abordar estos interrogantes, se diseñó un cuestionario on-line de 9 preguntas a la/os trabajadores de campo e investigadores del proyecto. Se trataba de analizar de qué manera afectaba a la investigación de campo y de qué forma podían adaptar las técnicas seleccionadas de recogida de datos (entrevistas narrativas, grupos de discusión y observaciones participantes) a la situación provocada por las medidas para controlar la epidemia en cada localización. Este artículo presenta los resultados del análisis de las respuestas al cuestionario virtual de las y los investigadores, complementadas con numerosas conversaciones virtuales para tratar de analizar cómo se producen los intercambios sociales en tiempos de distanciamiento físico³.

A partir de las respuestas obtenidas, el objetivo principal de este texto es analizar de qué manera se han visto afectadas y modificadas las relaciones entre investigadores y participantes. Además, se propone analizar el rol que está jugando el uso de los medios tecnológicos en este tipo de investigaciones etnográficas provocado por las medidas de confinamiento, en función de una variable fundamental en el proyecto debido a su temática: la confianza entre los investigadores y los jóvenes miembros de los grupos. Se trata de observar qué papel juega la confianza trabada durante el trabajo de campo físico en el uso de los medios virtuales para la continuidad de las actividades de investigación. En definitiva, proponemos un análisis de los cambios producidos en la comunicación entre investigadores y participantes en el proyecto (jóvenes, pero también stakeholders) pro- 
vocadas por el confinamiento, que ha obligado a un uso generalizado de formas de comunicación virtuales.

\section{2. "Home is the street"}

Una primera conclusión es que el mayor obstáculo para este tipo de investigaciones etnográficas han sido las medidas de distanciamiento físico instauradas por las autoridades de los países en los que se lleva a cabo la investigación: España, Italia, Francia, Marruecos, Túnez, Argelia, Estados Unidos, El Salvador, Cuba y Colombia. De manera generalizada, los diferentes gobiernos de las regiones de TRANSGANG han seguido, con matices, las mismas pautas: medidas sanitarias basadas en confinamientos de población; políticas de ayuda social con mayor o menor intensidad y éxito dependiendo de los recursos disponibles; mayor presencia policial y control de la calle, y mayor vigilancia por medios tecnológicos. Estas medidas de distanciamiento físico oscilan entre el confinamiento (es decir, la imposición de la obligación de quedarse en casa y restricciones en la movilidad con medidas represivas que van desde multas económicas hasta penas de reclusión) y la apelación a la responsabilidad individual sin medidas represivas a la transgresión de las normas. Lo que se propone es un control de la proxemia, es decir, de la distancia física, de las relaciones sociales establecida a través de lógicas culturales según el tipo de espacio donde se produce la relación entre individuos o grupos. La proxemia establece cuatro grandes tipos de espacios: íntimo, personal, social y público, según la distancia establecida entre los sujetos para sus relaciones (Hall, 1992), por tanto, el objeto de las medidas de distanciamiento social-físico pervierten y transforman las reglas sociales proxémicas establecidas consuetudinariamente. De hecho es paradójico el uso del término físico como adjetivo de distanciamiento, ya que parece obedecer a la idea de que, a pesar del distanciamiento físico obligado o recomendado, el mundo virtual salvaría el distanciamiento social provocado por las cuarentenas.

De esa manera, se impone un modelo de gobernanza donde la obsesión es el control de las movilidades y el contacto físico, convirtiendo en individuos peligrosos a aquellos que se ven obligados a seguir en la calle para ganarse el sustento diario $y$, en especial, a quien no tiene hogar. Asistimos de manera muy rápida a la implementación de una biopolítica de contención de los cuerpos en la cual el deambulante es un peligro para la salud pública y, en especial aquél que no tiene medios de subsistencia y está obligado a transgredir la nueva proxemia impuesta. Ya Foucault (2006) señalaba potencialmente, entre otras, dos cuestiones fundamentales para entender las medidas gubernamentales actuales: 1) el concepto de población para "evaluar" la eficacia de las medidas a través de "cifras" de infectados, casos graves y mortalidad (cuando una sola víctima ya es una tragedia); y 2) la marca del "transeúnte" como ser peligroso al no poder ser contado entre los miembros de la población sedentaria, pero también la consideración de cualquier persona deambulando por la calle como un "transgresor", convertida en delincuente social potencial. Así, son los sectores subalternos los más expuestos a la enfermedad y a las medidas represivas $y$, en especial, los grupos juveniles callejeros. Como señala Sihem Najjar, "esta categoría de jóvenes es la más expuesta al riesgo de contaminación porque viven en un entorno donde no se respeta el confinamiento. En el plano económico, sus condiciones socioeconómicas serán aún más críticas porque la mayoría de estos jóvenes (o sus familias) se encuentran en el sector informal, el cual tiene dificultades para funcionar durante el confinamiento"4 (Sihem Najjar, TRANSGANG, Túnez).

Los grupos callejeros que, por definición, consideran los espacios públicos -tanto virtuales como físicosun lugar central de convivencia, ya sea para obtener recursos de sustento o para socializar, están siendo obligados a transformar sus formas habituales de relación, con efectos más intensos y duraderos para ellos. Como señala Urteaga:

"el territorio de estos jóvenes había sido desplazado al ciberespacio, mientras que los investigadores se acercaban a ellos en el espacio convencional de la presencia, tratando de realizar etnografías, entrevistas, historias de vida a la vieja usanza, incapaces de sumergirse en el territorio donde la riqueza de las producciones lingüísticas, éticas y estéticas refulgía sin remilgos: blogs, foros y chats. A los investigadores les aparecía un nuevo territorio, que para esos jóvenes era el espacio natural de expresión" (Urteaga y Moreno, 2020, pág. 7).

Así, la tendencia de estos grupos a utilizar el ciberespacio como lugar de encuentro puede haber compensado estas limitaciones en relación a la comunicación, pero sigue siendo la calle el lugar central de convivencia y relación social para ellos. La calle, no el espacio público, sigue siendo el espacio central para ellos para resolver las carencias magnificadas por la situación actual. Así, durante la pandemia, hemos observado una mayor dependencia de las redes de apoyo no familiares, y al no poder acceder a ellas puede disminuir la capacidad de manejar la situación. Como señala nuestro investigador en Chicago, "se sienten conectados con el pulso de la calle, restringirlos a las paredes de su casa les hace sentir que no pueden escuchar sus latidos $y$, como tal, se están ahogando" (William D. Ross, TRANSGANG Chicago). En el mismo sentido afirma Margot Mecca, 
investigadora en antropología visual en el proyecto: "seguramente un aspecto que llama mucho la atención, en el estado de confinamiento, es la imposibilidad de acceder a un espacio público [la calle] que era espacio primario de socialización y que ahora está intensamente controlado por la policía." (Margot Mecca, TRANSGANG Barcelona). Por su parte Rachid Touhtou afirma para el caso marroquí: "para ellos, la calle es el hogar, por lo que el confinamiento no tiene significado ... la distancia social es realmente difícil para ellos ... comparten habitaciones pequeñas en viviendas sociales o en una ciudad pobre ... comparten los mismos canutos al fumar ... el grupo les proporciona apoyo, por lo que la distancia es un problema (...) Les está afectando ahora porque la calle es el centro de sus vidas y existencia ... les afectará primero el perder el apoyo de sus amigos" (Rachid Touhtou, TRANSGANG (asablanca). En este caso, para la/os jóvenes de grupos callejeros, distanciamiento físico significa aislamiento social, marginalización y precariedad.

Observamos una manera otra de entender los espacios públicos. Mientras los grupos juveniles entienden la calle como el lugar central de intercambios sociales donde se establecen confianzas y se traban lazos, también con la/os investigadores, las autoridades entienden la calle como un espacio público con la vocación de ser controlado para convertirlo en un espacio aséptico, forma de gobernanza hiperbolizada por la pandemia. Ahora es el momento de analizar de qué manera las relaciones entre participantes e investigadores trabadas en la calle influyen en la investigación virtual obligada por la situación provocada por la forma de gestionar la pandemia, y si las y los investigadores hemos superado estas barreras.

\section{Confianza, etnografía y redes sociales}

La encuesta realizada a nuestros investigadores e investigadoras revela cómo la irrupción de la pandemia provocada por el coronavirus ha transformado significativamente el campo de las relaciones entre los y las investigadores y sus informantes/participantes. Primero, el confinamiento establecido en las localizaciones ha dificultado la posibilidad de realizar trabajo de campo en el sentido clásico malinowskiano de la etnografía (Restrepo, 2015): recoger en el terreno de forma presencial, en estrecho contacto con las personas y los/las informantes, una información situada durante un tiempo prolongado. Resulta primordial que los y las antropólogos/as "debe(n) comparecer en el campo, reaprenderse y reaprender el mundo desde otra perspectiva" (Guber 2011, p. 50 en Contreras, 2016). Debido a la pandemia y el confinamiento ha sido imposible continuar con una de las técnicas clave de la antropología, la observación participante y no-participante, y seguir el campo y los contactos ya establecidos antes del confinamiento en base a unas relaciones de confianza entre investigadores e informantes (Contreras, 2016). Las relaciones presenciales establecidas a lo largo de un tiempo se vieron interrumpidas de forma más o menos brusca, sin poder preparar la salida temporal del campo de forma adecuada. Algunos de los contactos establecidos quedaron totalmente parados, según el lugar de residencia del/la joven y sus posibilidades de comunicarse con el mundo exterior, su grupo y la/os investigadores. María Oliver, investigadora en Madrid, constata: "Hago seguimiento a un joven que está en prisión. Estaba a punto de poder empezar a pedir permisos de fin de semana antes del confinamiento. Su última carta me llegó la semana del cierre de escuelas y yo le mandé una última ya en cuarentena. No he tenido más respuestas. Ahora sé de él a través de su madre, con la que me comunico por Whatsapp una vez a la semana." (María Oliver, TRANSGANG Madrid).

El paso obligatorio de la calle al hogar para la/os miembros grupos juveniles ha implicado también una transferencia hacia espacios más íntimos, los cuales en general son más difíciles de acceder y en tiempos de confinamiento casi imposibles: "Algunos de los jóvenes con los que trabajo no tendrían posibilidad de salir, así que la relación se mantendría en parte como hasta ahora, porque veo difícil ir a sus domicilios, en los que conviven con varios familiares, por ejemplo." (María Oliver, TRANSGANG Madrid). En el caso del grupo Debo, uno de los grupos participantes en la investigación en Túnez, entendieron que ese espacio íntimo y seguro era su sede, confinándose juntos allí y siguiendo con sus actividades habituales a pesar del distanciamiento físico. De todas formas, han sido las redes sociales las que han ofrecido nuevos o reforzados canales de comunicación con los y las jóvenes. Así, ha resultado relativamente fácil seguir sus actividades por Facebook (perfiles individuales y grupales) o sus canales de Youtube. Se ha mostrado que las comunicaciones y la información se produce en mensajes joven-investigador/a pero sin acceso directo a los canales de grupo. Lo clave aquí es tener el número de teléfono personal y que el joven tenga el número de la investigadora guardado, sin restricciones, de modo que se tiene acceso a las publicaciones privadas. De nuevo, la confianza trabada previamente físicamente continúa siendo fundamental. Paolo Grassi, investigador de Milán, afirma: "El ciberespacio es fundamental para contactar con estos jóvenes y seguirlos. Estoy usando en particular Whatsapp, Instagram y sigo a algunos de los raperos en Youtube. Las "historias" y el "estado" están dando información importante sobre la vida cotidiana [durante la pandemia] de mis interlocutores y sus imaginarios." (Paolo Grassi, TRANSGANG Milán). En 
los lugares con buena cobertura de internet y accesibilidad de jóvenes a dispositivos tecnológicos ha sido posible mantener con aquellos jóvenes con quienes ya se habían establecido unas relaciones de confianza una conversación fluida, informal, y cotidiana a través de canales como Whatsapp o Instagram ya que los mismos jóvenes utilizan de forma importante estos canales en su vida cotidiana y se sienten cómodos y familiarizados (a diferencia de lo que sucede con mucha/os investigadores). Como destaca María Oliver: "Considero que se relacionan muy fluidamente por redes sociales y se sienten en general cómodos hablando por mensajes, ya sea de texto o de audio, por lo que sólo a modo de diario de campo estoy encontrando que la conversación fluye y se puede hablar por amplios periodos de tiempo con ellos vía apps". (María Oliver, TRANSGANG Madrid).

Sin embargo, en regiones con mayores barreras tecnológicas, como en el Magreb o algunos países latinoamericanos, esta comunicación se ha dificultado de forma significativa. Se añade el hecho de que en lugares con regímenes autoritarios, debido al peligro de vigilancia y control del ciberespacio y las redes sociales, no resulta seguro para jóvenes comunicarse por estos canales. El investigador Anis Belgacem de Túnez observa: "Se llega a algunos de los jóvenes a través de las redes sociales, pero a menudo no es factible, por dos motivos: el primero es la posibilidad de que la entrevista se interrumpa o se detenga y (el segundo) es que a las personas que aún no te conocen les cuesta hablar libremente contigo a través de las redes sociales y responder tus preguntas, es bastante difícil."(Anis Belgacem, TRANSGANG Túnez). Cabe tener en cuenta que las conversaciones usando esos medios digitales que implican el registro de la voz y la imagen, generan para muchos jóvenes la sensación de ser más controlados, y menos informales y anónimas que en la calle. Los y las investigadores confirman que canales más "formales" como skype, zoom, etc. (de mayor uso por la comunidad académica y adulto/as en general) levantan una mayor sospecha entre informantes jóvenes. Así, los/as investigadores de TRANSGANG han optado a menudo por el uso de aquellos canales con los cuales sus informantes se sienten más cómodos, siempre y cuando se hubieran establecido unas relaciones de confianza basadas en el contacto físico antes del confinamiento. De esa manera, la posibilidad de continuar la comunicación a través de los nuevos canales de comunicación ha sido mayor cuando las relaciones de confianza ya se habían producido durante el período anterior a la pandemia. Al contrario, si no se había establecido esa confianza previamente, resulta más difícil mantener el contacto y reforzar la confianza, prueba de ello es la imposibilidad para los/as investigadores de establecer nuevos contactos con jóvenes durante la pandemia. Por otro lado, en un encuentro físico, según la investigadora Katia Núñez de Madrid "es más fácil captar gestos (lenguaje no verbal), por lo tanto creo que la cercanía física facilita más la confianza" (Katia Núñez, TRANSGANG Madrid). La realización de entrevistas de manera física proporciona esa información -que aporta datos fundamentales para una etnografía situada como la que propone TRANSGANG (Haraway, 1988)- contextual que se pierde en una relación virtual. Además, entender la etnografía como metodología exige un contraste entre el discurso y la práctica de los participantes, en especial en el caso de TRANSGANG por los temas tratados y, por lo tanto, en esta perspectiva las entrevistas virtuales pueden ser un recurso y no una estrategia. De todas formas, el reto de las etnografías virtuales consiste en entablar también en el espacio virtual unas relaciones de confianza desde una postura empática y de máximo respeto hacia los y las informantes y sus vidas privadas (Wijaya, 2012). La cuestión es cómo conseguirla; emprender una investigación etnográfica virtual exige un diseño, una planificación, unas cuestiones específicas relacionadas con el mundo virtual y unos objetivos así mismo específicos para esa forma de realizar etnografía. Podemos entrever que, por esto mismo, las dificultades provocadas por la pandemia en la etnografía propuesta (Feixa et al. 2019) son difíciles de solucionar, al no contemplar en el diseño inicial ese tipo de investigación.

Si atendemos a las redes sociales públicas, es cierto que a menudo favorecen una comunicación menos comprometida en el sentido de que permiten "esconderse detrás de un aparato", sobre todo cuando sólo es a través de texto. En cierta medida, las redes sociales públicas (muro de Facebook, Instagram, Twitter...) son un espacio público virtual que permite que tras el anonimato, como apunta Anis Belgacem de Túnez, sea "más fácil esquivar a alguien o ignorar a la persona en redes sociales que en la vida real" (Anis Belgacem, TRANSGANG Túnez), como reflejo de los lugares centrales de las urbes contemporáneas caracterizadas por poder pasar desapercibido. Por otro lado, las comunicaciones personales virtuales necesitan de trabar una confianza previa, convirtiéndose en un lugar semi-privado como la calle del barrio para ellos. Si te conocen puedes acceder, si falta esa confianza no podrás acceder. Esto muestra que el uso de este tipo de redes continúa, aunque bajo otras lógicas, siendo entendido de una manera similar a los encuentros en los espacios físicos. La ciudad anónima como los espacios virtuales de acceso ilimitado, y las redes sociales de uso personal e individual como el espacio privilegiado del barrio, donde para acceder tienes que ser reconocido como miembro de la comunidad. 
Por último, señalar que los y las investigadores se encuentran de forma general con la incógnita de sí podrán (re)conectar con jóvenes ya contactada/os y entrevistada/os. Esta cuestión se vuelve aún más pertinente en el caso de grupos juveniles con una alta movilidad geográfica, como el caso de jóvenes extutelada/os, provenientes de zonas magrebíes, residentes en las ciudades del Sur de Europa. Sin embargo, investigadores como Mustapha Omrane de Argel, afirman: "El grupo probablemente estaría dispuesto a programar nuevos encuentros después de que pase el encierro. Cuando pase el confinamiento, será fácil reunirse con grupos de jóvenes de la calle en los mismos lugares, no esperamos cambios en este aspecto." (Mustapha Omrane, TRANSGANG Argel) Estas expectativas se basan sobre todo en las buenas experiencias de haber mantenido unos lazos de confianza que perduran en el tiempo y en situaciones adversas como ésta de la pandemia.

\section{Trabajando el campo en tiempos pandémicos}

A partir del análisis de las respuestas extraídas de los cuestionarios hemos llegado a la conclusión de que la mayoría de investigadora/es locales consideran de vital importancia la relación de los miembros de los grupos, y de los grupos en su conjunto, con sus stakeholders de referencia. La percepción de cómo se retomarán o no estas relaciones tras el confinamiento varía en función de cada investigador/a, pero prácticamente todos coinciden en resaltar que la continuidad de las medidas de seguridad sanitaria, distanciamiento físico ${ }^{5}$ y control de aforo en los centros sociales y asociaciones en los que suelen encontrarse los grupos juveniles, pueden suponer un impedimento para el normal desarrollo de las actividades, y pueden alejar a la/os jóvenes de los centros y lanzarlos hacia las calles, parques y espacios abiertos en general, en los que no exista sobre ella/os la presión del cumplimiento de esas medidas. Juan Mansilla, investigador local de Marsella, percibe que "estarán más visibles en las calles que en las instituciones. Estas últimas estarán imponiendo más control para las reuniones grupales, lo que desmotivará, en principio, la vuelta de los jóvenes a dichas instituciones". (Juan Mansilla, TRANSGANG Marsella). Incluso si no se dieran las dificultades añadidas por la pandemia, la mera separación y pérdida de contacto por un espacio prolongado de tiempo ya supone una dificultad para las y los trabajadores de entidades sociales a la hora de retomar el contacto. Eduard Ballesté, investigador local de Barcelona, explica que "los distintos stakeholders que hacen trabajo con jóvenes deberán ampliar sus esfuerzos para volver a implicar a los jóvenes en sus actividades (...) Este tiempo, por lo que comentan los propios edu- cadores, puede generar un distanciamiento entre los jóvenes y las asociaciones." (Eduard Ballesté, TRANSGANG Barcelona). La desconfianza en el cumplimiento de las medidas de seguridad la encontramos también en alguna/os investigadores: Anis Belgacem, de Túnez, afirma "No me reuniría con ellos de inmediato ya que nada se habrá resuelto después de que pase el confinamiento y el distanciamiento social aún sería obligatorio. Todavía están activos en su estudio (...) y no me gustaría ir allí hasta que pase la tormenta de la COVID-19." (Anis Belgacem, TRANSGANG Túnez).

Por otro lado, conocer el estado de las relaciones inter-grupos es más complejo, aunque no imposible. Siguiendo a Zaki (2020), es acertado decir que las y los jóvenes, durante las fases más estrictas del confinamiento, han practicado esa "socialización a distancia" que, por otra parte, no soluciona los problemas provocados por distanciamiento físico, como son el apoyo mutuo y la solidaridad practicada por el propio grupo cotidianamente. Haciendo un seguimiento de sus redes sociales, ya sea en medios abiertos, como Youtube o grupos abiertos de Facebook, por ejemplo, como a través de Instagram o los estados de Whatsapp (aquí entra en juego la confianza previa establecida entre los investigadores y los jóvenes a la hora agregarles a sus redes sociales privadas), podemos hacernos una idea de cómo están manejando la situación.

Como hemos referido en el apartado anterior, en Túnez, Anis Belgacem afirma que el grupo Debo "siguen activos en su estudio, incluso durante este tiempo de confinamiento", (Anis Belgacem, TRANSGANG Túnez) y Paolo Grassi, en Milán, sigue a "alguno de los raperos en Youtube" (Paolo Grassi, TRANSGANG Milán); ambos investigadores proporcionan ejemplos de seguimiento de grupos musicales en redes sociales, y constatan que, ya sea juntándose en un mismo espacio físico o mediante grabaciones desde sus domicilios, los jóvenes de estos grupos siguen no sólo en contacto, sino creando, lo cual podemos entender como una forma de

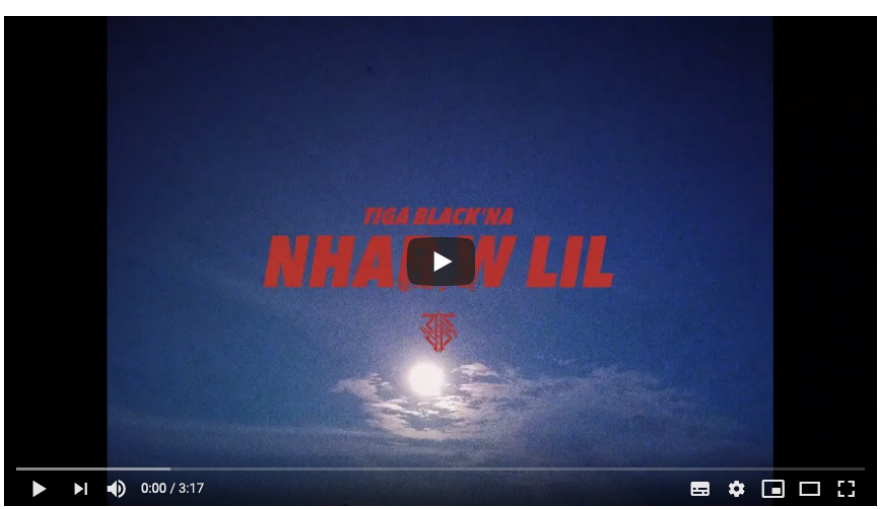

Figura 1. Videoclip realizado por el grupo DEBO. 
resistencia colectiva frente a las medidas gubernamentales impuestas.

No debemos perder de vista, en todo caso, que las medidas de confinamiento son distintas para cada uno de los casos aquí referenciados, y por lo tanto las estrategias de comunicación intergrupales dependen en parte de estas medidas, así como de las tradiciones socioculturales de sus respectivos contextos. Abel y McQueen (2020) hacen esta afirmación que encontramos que se aplica a los contextos del Magreb, por ejemplo:

En sociedades menos individualizadas que la mayoría de los países occidentales, el término "distanciamiento social" puede ser percibido como problemático y perturbador en contextos sociales y culturales en los que las personas están acostumbradas a recurrir las unas a las otras cuando los tiempos se ponen difíciles. A menudo, estos son países que son más propensos a sufrir la pandemia de COVID-19 debido a las malas condiciones basadas en economías débiles, infraestructuras débiles..." (Abel y McQueen, 2020, p. 231)

En Marruecos, Rachid Touhtou encuentra "señales de conexión online" (Rachid Touhtou, TRANSGANG Casablanca) y William Q. Ross, en EEUU, afirma que "hay un deseo creciente de los individuos por comunicarse" (William Q. Ross, TRANSGANG Chicago). Esta es, como decimos, la forma que tenemos de conocer el día a día de los grupos, a través de los miembros con los que podemos establecer contacto y "mirar" dentro de sus relaciones en redes. Paolo Grassi apunta a esta forma de seguimiento de los grupos: "Las "historias" y los "estados" están dando información importante sobre la vida cotidiana de mis interlocutores y sus imaginarios." (Paolo Grassi, TRANSGANG Milán).

Sabemos, por nuestra investigadora local en El Salvador, Cándida Chévez, que allí las comunicaciones por vía telefónica y telemática están controladas por el gobierno y cualquiera es susceptible de sufrir una intervención de sus comunicaciones. Así, los grupos, e incluso la/os stakeholders, en algunos casos, son precavidos en su uso: "En este momento no lo he utilizado debido a que las personas no sienten confianza ni seguridad de hablar abiertamente por medios virtuales de estos temas" (Cándida Chévez, TRANSGANG San Salvador). Sin embargo, al mismo tiempo, según informaciones de Cándica Chévez y de diversos medios de comunicación, los grupos se siguen organizando en sus respectivas zonas y barrios, e incluso entre dentro y fuera de las prisiones, con la dificultad añadida provocada por el aislamiento absoluto al que se ha sometido a los pandilleros en los centros penitenciarios. Aún así, las maras, tanto dentro como fuera de esos muros, parecen seguir una misma corriente, lo que hace suponer que mantienen abiertos ciertos canales de comunicación. La desconfianza en el uso de medios digitales para hablar de temas sensibles no la encontramos únicamente en El Salvador. Kammel Bourcherf explica que en Algeria "podemos afrontar muchas dificultades al usar Internet, por ejemplo el oficial de policía que no quiere mostrar su cara ni que se grabe su voz". (Kammel Bourcherf, TRANSGANG Algeria)

Encontramos, por lo tanto, que además de los problemas de conectividad y uso de las tecnologías, que podrían considerarse grandes impedimentos para el mantenimiento del trabajo de investigación, los diversos equipos locales afrontan un conflicto real en cuanto a la salvaguarda de la privacidad de los datos e información de carácter personal de las y los participantes en el proyecto que no deriva ni depende de las herramientas de investigación, sino de las medidas de control social de los Estados en los que se enmarca.

\section{Distanciamiento físico y control social: restricciones, conflictos y oportunidades}

Como hemos señalado, las medidas gubernamentales toman diferentes direcciones en cada país participante, siendo el distanciamiento físico con consecuencias sociales el objetivo general. El control social en tiempos de crisis se inscribe, antes que nada, en una lógica de reglamentación nacional, con leyes que enmarcan las medidas particulares impuestas en las ciudades, e incluso en barrios específicos. Por supuesto, dichas medidas gubernamentales no solo responden a las posibilidades normativas existentes, sino que emergen dentro de la coyuntura económica, política y tecno-social de cada país. Entender los efectos del control social desde la perspectiva de sociedades neoliberales, exige no solo considerar dichas coyunturas dentro de cada territorio, sino entender las lógicas entre democracia y autoritarismo que operan en cada uno de ellos. En países con "democracias delegativas" (García, 2012 p. 20), donde el voto popular existe, pero con poderes públicos que facilitan los intereses partidistas del ejecutivo y de las oligarquías económicas, como las latinoamericanas o magrebíes, "la forma como se encuentra estructurada la distribución del poder, tiene mayor tendencia a brotes de autoritarismo y polarización política" (Linz y Valenzuela, 1994, p. 44). En estos países, basados en regímenes presidencialistas y monárquicos - herencia del colonialismo europeo - con democracias aparentemente representativas, el control social durante las crisis, de cualquier índole, se usa para silenciar, desde la legalidad o la paralegalidad, las acciones divergentes al 
gobierno de turno, como sucede, también, con el control de la pandemia.

Los jóvenes de grupos callejeros en las diversas localizaciones del proyecto han continuado, en mayor o menor medida y a pesar de las medidas de confinamiento, ocupando espacios públicos y reuniéndose en grupos. Esto ha traído consigo un aumento considerable de confrontaciones entre estos jóvenes y las fuerzas policiales. De manera general, la persecución policial a la/os jóvenes de grupos callejeros ha aumentado en cada uno de los países estudiados. María Oliver, investigadora en Madrid, afirma que una de las razones ha sido que "al haber menos gente por la calle, [los jóvenes] son más visibles cuando salen" (María Oliver, TRANSGANG Madrid). Las represiones policiales violentas contra la/os jóvenes han sido señaladas en Francia (principalmente en la región parisina), en Barcelona y en El Salvador. Al norte de París, en Villeneuve-la-Garenne una ciudad con $41 \%$ de habitantes menores de 25 años y donde $60 \%$ de las residencias son viviendas de carácter social - un joven motociclista fue gravemente herido durante una colisión con un coche de policía que no tenía distintivos institucionales. La noticia se propaga rápidamente por redes sociales. La policía afirma que el joven quiso huir de un control de identidad, un desafortunado accidente. Los testigos contradicen la versión, un policía abrió la puerta del coche de manera intencional, sin mediar palabra, haciendo caer al joven que iba a gran velocidad en la moto, y sin casco. La noche siguiente, decenas de jóvenes indignados toman las calles, no solo del barrio, sino de barrios vecinos, sin importar las medidas de confinamiento y sus consecuencias. "Los jóvenes dispararon morteros, fuegos artificiales y prendieron fuego a botes de basura y automóviles. A cambio, recibieron gases lacrimógenos", publica el diario Liberation.

En Chicago, tras la muerte del afroamericano George Floyd, el 25 de mayo del 2020, asfixiado en el suelo por un policía, el movimiento Black Lives Matters (BLM) de protestas callejeras se propaga rápidamente por todo Estados Unidos. Centenares de personas ocupan las calles de Chicago para denunciar la brutalidad policial y el racismo sistemático. BLM es respaldado en diferentes ciudades en el mundo, cada una tiene su propio George Floyd, su propia lucha contra la violencia policial. En Francia, el 2 de junio, y a pesar de las prohibiciones de reuniones masivas en espacios públicos por la crisis sanitaria, emerge una protesta masiva "antiracista" contra la brutalidad policial denunciando la muerte en 2016, a manos de policías, de Adama Traoré, un joven negro habitante de la banlieue norte parisina. Estos conflictos entre las fuerzas policiales y las poblaciones más vulnerables se han producido a pesar de las medidas de restricción de movilidad y desencadenado movimientos masivos de protesta en las calles, a pesar del confinamiento y las consecuencias punitivas y sanitarias que ello implica. ${ }^{6}$

Por otra parte, el aumento del control de la movilidad transnacional ha sido también un abanderado de las medidas restrictivas en tiempos de pandemia. Muchos países, frente al brote epidémico, han implementado prohibiciones de entrada de personas no-nacionales en sus territorios, imponiendo cuarentenas obligatorias para todo viajero, o cerrando fronteras. En algunos países TRANSGANG, el control social gubernamental ha sido ejercido de manera coercitiva. Las medidas extraordinarias de control social estatal han sido la militarización del espacio público, las penas pecuniarias y hasta las penas de prisión. Se han utilizado generalmente sanciones pecuniarias que, en ciertos casos, pueden llegar a ser reemplazadas por penas privativas de la libertad, como es el caso de Argelia, Túnez, Marruecos y El Salvador.

Los gobiernos utilizan diferentes canales de comunicación para difundir los mensajes de control social a la población. En Chicago, por ejemplo, las actualizaciones de las medidas son notificadas a la población "a través de mensajes en carreteras, aeropuertos, en el sitio web de la Ciudad y en las redes sociales". Igualmente, por orden administrativa, los comerciantes sirven de puntos de relevo sobre las últimas actualizaciones de información de control social: "se solicitará a los hoteles y alquileres a corto plazo que comuniquen la cuarentena de 14 días a los huéspedes que hayan viajado desde uno de los estados designados".7 En Marruecos, se lanzan mensajes utilizando vehículos adaptados con grandes megáfonos que circulan por los barrios periféricos alentando a la población a que se quede en su casa. A pesar de ello, en los barrios periféricos e informales de las ciudades argelinas, tunecinas y marroquíes, cuyas poblaciones entienden el barrio como un lugar semi-privado de relación social con sus vecinos, continúan las actividades de economía informal que permiten la subsistencia de poblaciones muchas veces olvidadas por los beneficios sociales.

Incluso, como señala Kamel Bourcherf, investigador en Argelia, "Aquí hay grupos que venden productos en la calle y cuando se les preguntó sobre su permanencia en la calle, su respuesta fue que prefieren ser afectados por el virus e ir al hospital para obtener el apoyo estatal. Hay grupos que se aislaron y ya no lo hacen. Otros grupos no parecen evitar la Covid-19 sino evitar a la policía reuniéndose muy tarde en la noche." (Kamel Bourcherf, TRANSGANG Argelia). Como vemos, el riesgo de sanción por incumplimiento de las medidas de distanciamiento 


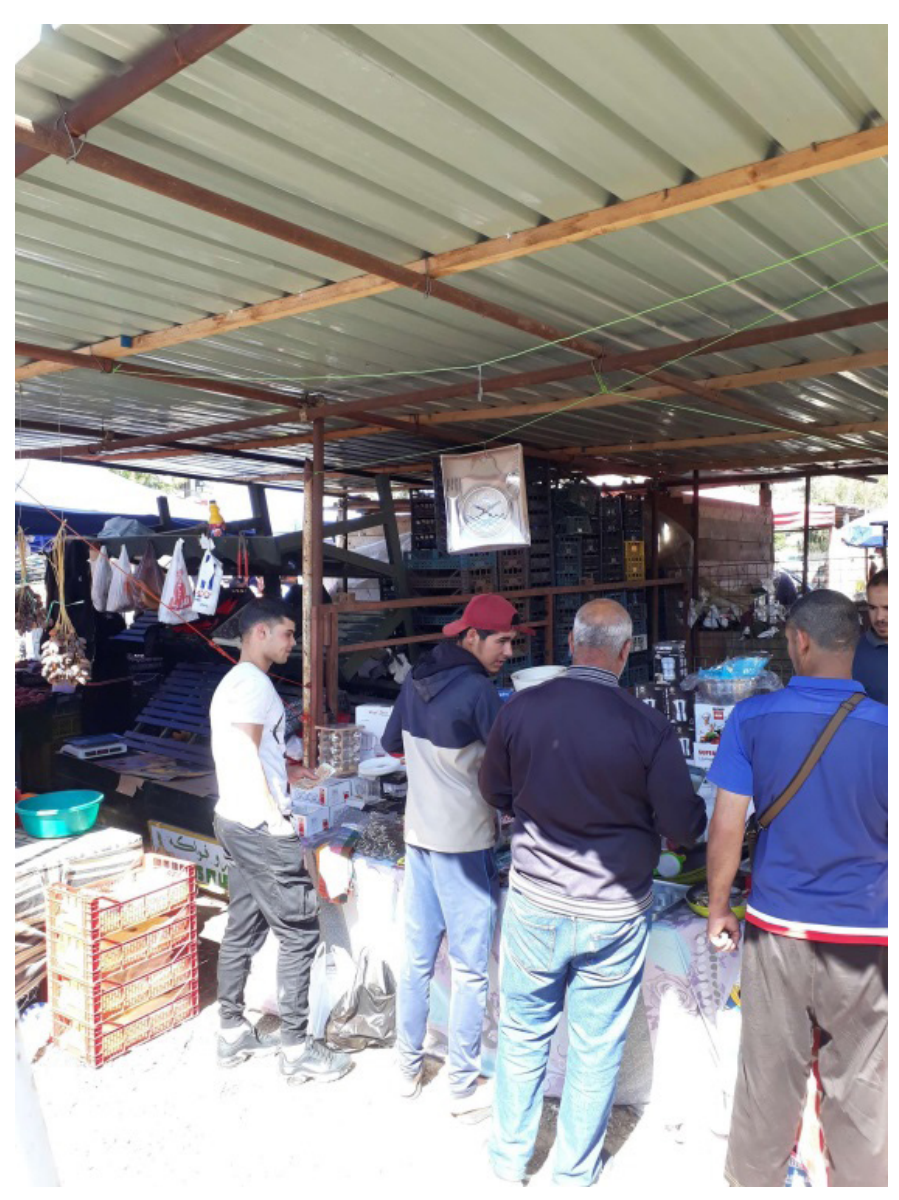

Figura 2. Durante los meses de confinamiento, los habitantes del distrito de Beni-Messous (Argel) han estado comprando sus frutas y verduras en este mercado construido en un espacio reservado para el aparcamiento de autobuses habitualmente. (Foto: Mustapha Omrane, Investigador TRANSGANG en Argelia).

físico se sopesa de manera diferente en cada país analizado, con múltiples variables en juego que escapan al objetivo de este artículo. Sin embargo, es necesario señalar que el confinamiento representa para las poblaciones más pobres de estos países una amenaza inminente para sus vidas, incluso más certera que la misma pandemia.

Siendo los jóvenes de grupos callejeros poblaciones históricamente marginalizadas, viviendo de redes de solidaridad vecinal y de economía informal, su principal estrategia de supervivencia durante el confinamiento ha consistido en encontrar formas innovadoras de continuar ocupando la calle y alimentado dichas redes de solidaridad - de manera virtual, gracias a internet, pero sobre todo de manera presencial, pues de ello depende el acceso a recursos materiales como la comida y el dinero. En Marruecos (Casablanca), una forma de resistencia al confinamiento es la venta informal en las calles, lo que implica nuevas formas de negociación del espacio público entre la población y las autoridades, como señala el investigador Rachid Touhtou, "desafían a las autoridades saliendo y tratando de ganarse la vida ... algunos de ellos corren el riesgo de ir a la cárcel ... para ellos, la calle es su hogar, así que el confinamiento no tiene sentido ... la distancia social es realmente difícil para ellos". (Rachid Touhtou, TRANSGANG Marruecos). En Francia, el tráfico de drogas al menudeo ha continuado durante el confinamiento. "Los grupos que se dedican a ello [tráfico de drogas al menudeo], juveniles o no, utilizan cada vez más las redes sociales digitales y la entrega a domicilio (u otras formas creativas) para continuar estas actividades", señala Juan Mansilla, investigador en Marsella.

Ante el aumento del control social, justificado por las medidas excepcionales de lucha contra la reciente pandemia, la/os jóvenes de grupos callejeros han recurrido a nuevas formas de relacionarse entre ella/os y con sus comunidades. Entre el confinamiento y las salidas no autorizadas a los espacios públicos, estos grupos se adaptan a las nuevas condiciones de control social. Como afirma María Oliver, estos grupos "están acostumbrados a pasar tiempo separados por diferentes motivos (prisión, presión policial, alejamientos temporales), pero tienen facilidad para volver a juntarse y retomar actividades" (María Oliver, TRANSGANG Madrid). Otro aspecto fundamental es el conflicto histórico de esta/os jóvenes con las instituciones que, en cierta medida, reproducen las escalas de marginalidad que ellas mismas denuncian. La adaptación y la resistencia se convierten así en formas innovadoras de vivir el nuevo control social. Cierto, la obediencia aparente de estos grupos a las medidas de distanciamiento físico les evita confrontaciones con la policía. Sin embargo, su aplicación efectiva - como el porte de mascarillas, el no contacto físico y el mismo confinamiento - "son considerados como símbolos de debilidad entre los miembros de estos grupos", y en consecuencia no las cumplen, como señala William Q. Ross, investigador en Chicago. Esta llamada debilidad hace referencia a una interdependencia que enlaza no sólo a los miembros del mismo grupo y a estos con otros grupos rivales, sino a las juventudes marginalizadas con gobiernos más o menos autoritarios y represivos. En este sentido, una parte de la población en los países analizados no considera que la pandemia reciente constituya una amenaza real. En consecuencia, toda medida gubernamental fundada en la pandemia les resulta ilegítima aunque reconocen la labor de sanitarios y difunden las medidas sanitarias pintando murales en los barrios para que la población analfabeta o analfabeta instrumental conozca la tríada sanitaria: mascarilla, manos y distancia. El grafiti, otrora criminalizado por su poder de comunicación de discursos alternativos, se convierte en un medio de 
comunicación fundamental para las poblaciones marginalizadas de eso barrios periféricos (Laine, 2018).

Sin embargo, el escepticismo se alimenta del olvido histórico del que han sido objeto por parte de innumerables gobiernos que, en el mejor de los casos les ignoran, y en el peor usan sus vidas como punta de lanza en conflictos internos. En El Salvador, el gobierno actual es acusado por varios medios de utilizar, durante el confinamiento, a una de las pandillas para eliminar a las otras. En Colombia, y varios países latinoamericanos, el juvenicidio (Valenzuela, 2015), como fenómeno de precarización y estigmatización de las conductas juveniles, hace aún más frágiles los escenarios de libertad social.

\section{Algunas reflexiones finales}

A partir de nuestros análisis podemos establecer algunas conclusiones generales relacionadas con las metodologías etnográficas y apuntar algunas cuestiones que serán fundamentales para los próximos meses de investigación.

En primer lugar, las y los investigadores tendrán, forzosamente, que rediseñar sus planes y calendarios de investigación para adaptarlos a la nueva temporalización, condicionada por la pandemia. Esa adaptación deberá tener en cuenta la posibilidad de que las medidas de confinamiento y/o restricciones a la movilidad se repitan en un futuro cercano. La dificultad estriba en adaptar investigaciones planeadas para la ser llevadas a cabo en espacios físicos para realizarse a través de medios virtuales. Como hemos visto, eso es posible cuando se ha trabado la necesaria confianza previamente, sobretodo en investigaciones sobre temas sensibles como la planteada por el proyecto TRANSGANG. Por eso, para la adaptación, uno de los objetivos primeros será el afianzamiento de las relaciones de confianza con las y los jóvenes y stakeholders, de modo que incluso en el caso de nuevos confinamientos se pudieran mantener contactos por medios digitales de forma más fluida garantizando siempre la confidencialidad de las y los participantes. La dificultad mayor, en estados autoritarios, estriba en superar la desconfianza generalizada tanto de miembros como de stakeholders con los medios virtuales, y superar los condicionamientos tecnológicos en lugares que sufren una pobreza tecnológica. De ahí que la confianza sea uno de los mayores capitales que han podido ir acumulando los y las investigadoras del proyecto durante estos meses y que ha permitido seguir realizando algunas actividades de campo.

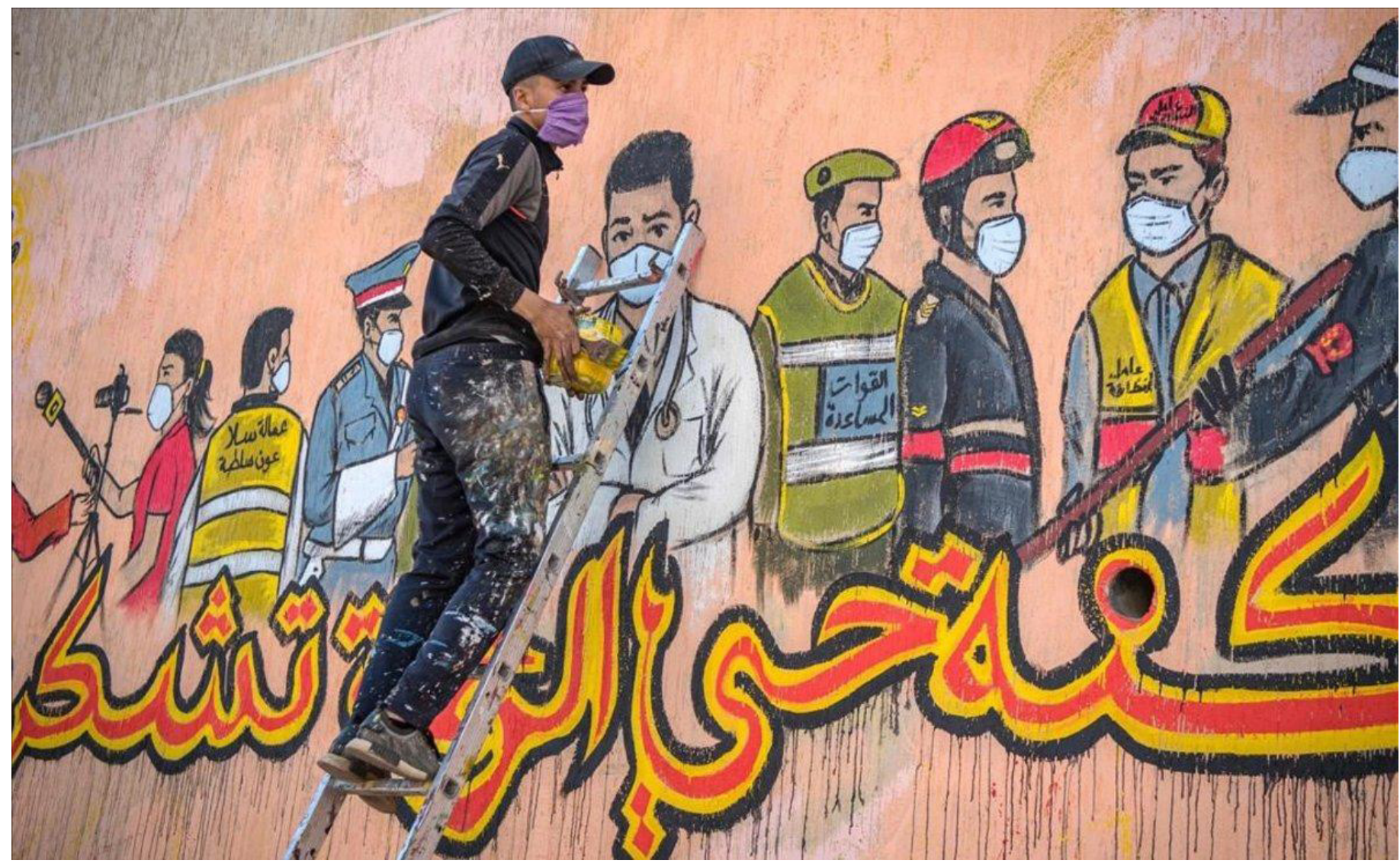

Figura 3. Un artista local pinta un mural dedicado a los trabajadores de sectores esenciales durante el confinamiento en Salé, localización del proyecto TRANSGANG (Marruecos). Fuente: El Periódico. 
Por otra parte, siguiendo con el análisis realizado, los espacios públicos virtuales se han convertido en un lugar fundamental para asegurar la comunicación durante la pandemia entre la membresía de manera aparentemente segura y anónima, de forma similar a los intercambios comunicativos que pueden producirse en los centros de las ciudades. Sin embargo, para los grupos juveniles, como hemos observado, la calle continúa percibiéndose como espacio fundamental para desarrollar sus actividades, obligados a la transgresión de las medidas de confinamiento por necesidades materiales. En este sentido, como una unidad social, en el vecindario, la membresía en la comunidad es el mecanismo clave para la relación social central para instituciones como las pandillas y las asociaciones económicas informales. Para llegar a investigar en esos espacios físicos es necesario trabar redes de confidencialidad que permitan la intrusión del etnógrafo en la vida cotidiana. Igualmente, el uso de las comunicaciones virtuales con los miembros de los grupos para el trabajo de campo necesita de trabar confianzas previas para acceder a esa información. Como vemos, las redes sociales para la comunicación individual también necesitan de la confianza previa establecida en los contactos físicos que permiten el emplazamiento del investigador/a en su territorio "privado", ya sea físico o virtual. ${ }^{8}$

Finalmente, cabe preguntarse, a manera de proposición para nuevas investigaciones, ¿cómo el nuevo control social propio de lo que se ha denominado "nueva realidad" afectará a los grupos juveniles de calle? ¿Qué implicaciones tiene el control social por distanciamiento físico en las lógicas callejeras de los jóvenes de barrios marginalizados?

\section{Financiación}

Este artículo es uno de los resultados del proyecto TRANSGANG que ha recibido financiación del European Research Council (ERC) en el marco del programa de Investigación e Innovación de la Unión Europea HORIZON 2020, grant agreement No 742705. Queremos también agradecer a los investigadores/as del proyecto la disposición y compromiso con el que han participado en la recolección de datos que ha permitido realizar este artículo. Para mayor información puede consultarse la página web del proyecto: https://www.upf.edu/web/ transgang. También puede verse el video de presentación de la investigación en el canal de Youtube de TRANSGANG: https://www.youtube.com/watch?v=Vbs02ur9lwlet $=8$ s

\section{Notas al final}

1. El grupo DEBO adopta una posición antisistema y, sobre todo, un interés por los problemas y preocupaciones de los jóvenes marginados cuyos derechos reivindica a través de la expresión artística. DEBO está desempeñando un papel mediador al ubicar sus actividades y eventos en un marco institucional, logrando dar a conocer el underground y por ende lo ilegal; e intentando visibilizar prácticas que son consideradas o ilegales en Túnez (el grafiti, la actitud antisistema, las drogas, el discurso violento, etc.), pero en su mayor parte legitimadas socialmente entre los grupos juveniles de calle tunecinos. La casa Kolacho en la Comuna 13 de Medellín se ha convertido en un centro de mediación en una de las zonas más violenta de la ciudad. Fue fundada por miembros y ex miembros de grupos juveniles para cambiar la violenta realidad del barrio. Se ha convertido en un centro referencial, como punto de encuentro de artistas urbanos que enseñan a los jóvenes del barrio una alternativa para no caer en la criminalidad

2. Siguiendo a Restrepo, "la etnografía como metodología, como encuadre, estaría definida por el énfasis en la descripción y en las interpretaciones situadas. Como metodología, la etnografía buscaría ofrecer una descripción de determinados aspectos de la vida social teniendo en consideración los significados asociados por los propios actores. Esto hace que la etnografía sea siempre un conocimiento situado; en principio da cuenta de unas cosas para una gente concreta" (Restrepo, 2015: pág. 163).

3. El cuestionario, enviado a las y los investigadores a principios del mes de abril, contenía ocho cuestiones que abordaban tanto la situación de los grupos juveniles en cada localización TRANSGANG como sobre la afectación provocada por las medidas de confinamiento en el trabajo de campo y una pregunta abierta final. Se obtuvieron 16 participantes provenientes de las tres regiones representadas en el proyecto, Magreb (Marruecos, Argelia y Túnez), del Sur de Europa (España, Francia e Italia) y América (El Salvador, Colombia y Estados Unidos). Enlace al cuestionario enviado en inglés y castellano a la/os investigadores: https://docs.google.com/forms/ d/1TB6Lw7miU7dYHkm7fFpZJOjnDnH4IE5A36R1aGSQSSis/ edit?usp=drive_web

4. Las respuestas de la/os investigadoras/es del Norte de África, Italia y EEUU se proporcionaron en inglés y se traducen al castellano para este artículo.

5. Aunque en estos meses se haya normalizado el uso de la expresión "distanciamiento social", consideramos más acertado el término "distanciamiento físico": Para empezar, "distanciamiento social" era el término equivocado. Deberíamos pensar en este momento como un "distanciamiento físico" para enfatizar que podemos permanecer socialmente conectados incluso estando separados. De hecho, los animo a todos a practicar la "socialización a distancia". (Zaki, 2020) https://news. stanford.edu/2020/03/19/try-distant-socializing-instead/

6. Para mayor información véase el video: "'Black Lives Matter" : George Floyd, Adama Traoré... Paris manifeste à son tour" https://www.youtube.com/watch?v=IMwnNC-vad8

7. Public Health Order No. 2020-10: Phase III: Quarantine Restrictions on Persons Entering Chicago from High Case-Rate States: https://www.chicago.gov/city/en/ sites/covid-19/home/emergency-travel-order.html

8. Como hemos indicado anteriormente, otra cuestión es el diseño de una investigación basada únicamente en una etnografía virtual que se planifica estrategicamente para conseguir esa confidencialidad necesaria para el acceso a las comunicaciones virtuales. 


\section{Referencias}

Abel, T., y McQueen, D. (2020). The COVID-19 pandemic calls for spatial distancing and social closeness: not for social distancing. Int $\mathrm{J}$ Public Health, 65, 231.

Contreras, P., Assaél, J., Acuña, F., Santa, E., Campillay, B., y Pujadas, B. (2016). Construyendo saber etnográfico: Reflexiones a partir de la experiencia de campo en instituciones escolares. Athenea Digital, 16(3), 55-79.

De Witte, M. (19/03/2020) Instead of social distancing, practice "distant socializing" instead, urges Stanford psychologist, Standford News. https://news.stanford.edu/2020/03/19/try-distant-socializing-instead/

Feixa, C., Sánchez García, J., Ballesté, E., Cano, A. B., Masanet, M.-J., Mecca, M., y Oliver, M. (2019). La (Trans) Banda: Notas y cuestiones para la investigación con grupos juveniles de calle. Universitat Pompeu Fabra y European Research Council. https://doi. org/10.31009/transgang.2019.wp02.2

Feixa, C., Sánchez-García, J., y Brisley, A. (2020). "Gangs, Methodology, and Ethical Protocols: Ethnographic challenges in researching youth street groups". Journal of Applied Youth Studies, 1-16. https://doi. org/10.1007/s43151-020-00009-1.

Foucault, M. (2006). Seguridad, Territorio, Población. Curso en el College de France, 1977-1978. Fondo de Cultura Económica

García Oñoro, J. (2012). Latinoamérica: entre la democracia y el autoritarismo, 41. https://core.ac.uk/display/120122373

Hall, E. (1992). An Anthropology of Everyday Life: An Autobiography. Anchor Books

Haraway, D. (1988). Situated Knowledges: The Science Question in Feminism and the Privilege of Partial Perspective. Feminist Studies, 14(3), 575. https://doi.org/10.2307/3178066

Laine, S. (2018). 'Todavía Tenemos Muros en los que Pintar'. De la Iniciativa de Cinco Jóvenes al Movimiento Grafiti Global. Estudio de Caso de Zwewla (Miserables). En Sánchez García, J., Ballesté, E., y Feixa, C. (eds.) ¿Qué fue de la primavera indignada? Movimientos sociales política y juventud en tres continentes. Milenio

Linz, J. J., y Valenzuela, A. C. N.-J. . F. 1994. (1994). The failure of presidential democracy. Johns Hopkins University Press.

Restrepo, E. 2015. El proceso de investigación etnográfica: Consideraciones éticas. Etnografías Contemporáneas 1 (1), 162-179.
Urteaga, M., y H.C. Moreno. (2020). Jóvenes mexicanos: violencias estructurales y criminalización. Revista de Estudios Sociales 73

Valenzuela, J.-M. (2015). Juvenicidio: Ayotzinapa y las vidas precarias en América Latina. El Colego de la Frontera Norte.

Wijaya, S. W., Watson, J., y Bruce, C. (2012). Addressing Public and Private Issues in a Virtual Ethnography Study of an Open Online Community: a Reflective Paper. Queensland University of Technology ePrints Archive. http://eprints.qut.edu.au/61858/

\section{CV}

Jose Sánchez García. Es doctor en Antropología Social y Cultural por la Universidad de Barcelona. Actualmente es Coordinador Científico del proyecto TRANSGANG.

María Oliver. Es investigadora predoctoral en la Universitat Pompeu Fabra de Barcelona. Antigua miembro de una pandila, actualmente es Investigadora Local del proyecto TRANSGANG en Madrid.

Juan Camilo Mansilla. Tiene un doctorado en ciencias de la información y la comunicación de la Universidad Sorbonne Paris Cité. Actualmente es Investigador Local en Marsella y Coordinador Transmedia de Gangpedia Space en TRANSGANG.

Nele Hansen. Es doctora en Antropología Social y Cultural por la Universidad Autónoma de Barcelona (UAB). Actualmente es Project Manager del proyecto de investigación ERC TRANSGANG.

Carles Feixa. Es catedrático de antropología social en la Universitat Pompeu Fabra de Barcelona. Actualmente es el Investigador Principal del proyecto de investigación TRANSGANG.

\section{осм Observatorio de Cibermedios}

\section{https://observatoriocibermedios.upf.edu/}
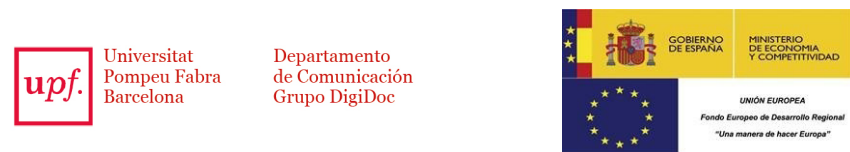

El Observatorio de Cibermedios es una producción del Grupo de Investigación en Documentación Digital y Comunicación Interactiva (DigiDoc) del Departamento de Comunicación de la Universitat Pompeu Fabra.

El Observatorio de Cibermedios (OCM) forma parte del proyecto del Plan Nacional "Narración interactiva y visibilidad digital en el documental interactivo y el periodismo estructurado". RTI2018-095714-B-C21 (MINECO/FEDER), Ministerio de Ciencia, Innovación y Universidades (España). 